\section{Testosterone concentrations in 37 dogs: breed differences and the effect of passive smoke exposure}

\section{Clare Knottenbelt, Natalie Hutchinson, Dominic Mellor}

College of Medical, Veterinary and Life Sciences, University of Glasgow, Glasgow, Scotland, UK

\section{INTRODUCTION}

Testosterone concentrations ( $\mathrm{T}$ ) are important in determining dominance behaviours in humans. In dogs castration changes behaviours in 50-60\% of dogs (increased bodyweight, increased appetite and decreased activity). Chronic direct smoking reduces canine $T$ significantly, however the impact of environmental tobacco smoke (ETS) exposure on canine health is currently poorly characterised. Hair nicotine concentration $(\mathrm{HNC})$ is an accurate method of determining ETS exposure in dogs. The data presented is part of larger study to assess the impact of ETS exposure on ageing markers in pet dogs in a home environment. The study has been approved by the local Ethics and Welfare committee.

\section{MATERIALS AND METHODS}

37 entire male dogs were recruited and underwent physical examination (including body weight and condition score) and routine bloods prior to and 12 months post routine castration. 24 dogs were reassessed at 12 months post castration. T was measured in discarded plasma by ELISA (T1 and T2). HNC was measured using LC-MS/MS at both time points.

\section{RESULTS}

The dogs comprised 7 Staffordshire bull terrier (SBT), 2 SBT crossbreds, 7 non-SBT crossbreds, 4 Border collies, 2 CKC spaniels, 2 Yorkshire Terriers and 2 Springer spaniels. Only 2 dogs in the SBT group returned for the 12 month check. Ages ranged from 5.5 months -7.5 years (median $=11.5$ months). Pre-castration $T$ ranged from $1.0 \mathrm{nmol} / \mathrm{l}$ to $\geq 56$ $\mathrm{nmol} / \mathrm{l}$ (median $=43 \mathrm{nmol} / \mathrm{l})$. Neither T1 or T2 correlated with age or weight. T1 was significantly higher in SBT and SBT crosses when compared to the other breeds $(p=0.001)$. $T 1$ was significantly lower in pre-pubescent dogs compared to pubescent and post-pubescent dogs $(p=0.04)$.

T2 ranged from $0.72-2.43 \mathrm{nmol} / \mathrm{I}$ (median $=1.71 \mathrm{nmol} / \mathrm{I})$ and was no associated with T1. T2 was inversely associated with increased weight in 24 dogs over the 12 month period (p 0.028, R2=0.20). Although there was no association between $\mathrm{T}$ and $\mathrm{HNC}$ at either time point, proportional weight gain at 12 months post castration was significantly associated with $\mathrm{HNC}(\mathrm{p}=0.03, \mathrm{R} 2=0.20)$.

\section{CONCLUSIONS}

SBT and SBT crosses have higher pre-castration $T$ which could explain their breed associated behavioural traits. T levels are not associated with ETS exposure as measured by $\mathrm{HNC}$, however weight gain post castration may be affected by both ETS exposure and lower post-castration $\mathrm{T}$ (which is not associated with T1).

\section{Clinical and clinicopathological features in dogs with recently diagnosed uncomplicated spontaneous hyperadrenocorticism in first opinion practice (2013-2014)}

\section{Michael Bennaim ${ }^{3}$, Steven Centola ${ }^{2}$, Ian Ramsey ${ }^{2}$, Mayank Seth'}

\footnotetext{
1 Animal Health Trust, Kentford, Newmarket, Suffolk, UK

2 University of Glasgow, Faculty of Veterinary Medicine, Glasgow, Scotland, UK

3 University College Dublin, Faculty of Veterinary Medicine, Belfield, Co. Dublin, Ireland
}

Clinical signs and clinicopathological findings of spontaneous hyperadrenocorticism (HAC) were reported several decades ago. Since that time several reviews and texts have commented that large breeds of dogs with HAC show less clinical features than smaller breeds and that the prevalence of various signs may be lower nowadays, with diagnosis made at earlier stages.

An online questionnaire was made available to veterinarians in the United Kingdom and the United States to gather information pertaining to signalment, clinical signs, physical examination findings and clinicopathological abnormalities of dogs diagnosed with HAC in first-opinion practices. Inclusion criteria were an ACTH stimulation test and/or low-dose dexamethasone suppression test consistent with HAC. Exclusion criteria included known concurrent systemic diseases or any sign not typically associated with HAC (e.g., vomiting, diarrhoea), a low urinary cortisol:creatinine ratio, possible administration of steroids over the 3 month before diagnosis or treatment with a drug known to cause the investigated clinical signs. For each case, a clinical signs ratio (CSR) was calculated for the number of clinical signs present out of the total number reported to have been assessed in that case.

Sixty-two cases were identified. The prevalences of clinical signs and clinicopathological abnormalities were similar to previously reported. There was no correlation between the weight and the CSR and no difference between the CSR in small breeds $(<20 \mathrm{~kg})(\mathrm{n}=40)$ and large breeds $(>20 \mathrm{~kg})$ $(n=21)$. The prevalence of nocturia, polyphagia, abdominal enlargement, alopecia, hyperpigmentation, recurrent pyoderma, lethargy, excessive panting, muscle weakness and muscle atrophy were not statistically different in large breeds and smaller breeds. The platelet count was negatively correlated to the weight $(p=0.005)$. Alanine aminotranferase (ALT $(p=0.016, \quad r 2=0.17)$ and alkaline phosphatase (ALKP) $(p=0.05, r 2=0.0014)$ activities were both positively correlated to the CSR. 
In this group of dogs, the range of clinical signs is not significantly different between small and large breeds. The prevalences of clinical and clinicopathological findings appears to be similar to those historically reported. Dogs with more clinical signs tend to have higher ALT and ALP activities.

\section{Canine Hba1c: validation of an immunoturbidimetric assay and determination of a reference interval}

\section{Anne Florentine Goemans, lan Ramsey}

University of Glasgow, Glasgow, UK

\section{BACKGROUND}

Haemoglobin A1c ( $\mathrm{HbA1c})$, provides a reliable measure of glycaemic control over 2 to 3 months in human diabetics. In dogs, formation of $\mathrm{HbA1c}$ has been demonstrated; but currently no commercial assays exist for determining canine $\mathrm{HbA1c}$. The validation of such an assay could have significant benefits for the management of canine diabetes.

\section{OBJECTIVE}

To validate a commercially available automated immunoturbidimetric assay for canine $\mathrm{HbA1c}$ and determine a reference interval for canine $\mathrm{HbA1c}$ in a normoglycaemic hospital population.

\section{METHODS}

The specificity of the assay was assessed by inducing $\mathrm{HbA1c}$ in vitro using isolated canine haemoglobin preparations. Imprecision was assessed using manufacturer supplied control materials for between run imprecision. The linearity was assessed by mixing known samples in differing proportions and the effect of anticoagulants by taking paired samples. 7 Samples with known lipid concentrations, triglycerides from $0.68-25.58 \mathrm{mmol} / \mathrm{L}$ and cholesterol from 4.9-11.7 $\mathrm{mmol} / \mathrm{L}$ were measured before and after removal of serum and replacement with $0.9 \%$ saline. The effect of haemolysis was also determined. A reference interval and the effect of age and breed predisposition was determined using EDTA whole blood samples from 60 normoglycaemic non-anaemic hospitalised animals. Measured values were expressed in terms of mmol of $\mathrm{HbA1c}$ per mol of haemoglobin.

\section{RESULTS}

$\mathrm{HbA1c}$ increased proportionally with glucose concentration in vitro. The between run imprecision was 5.9\% (3\% and 8.8\%). The assay was linear within the expected working range. EDTA and heparin can be used interchangeably for $\mathrm{HbA1c}$ measurement. Measured $\mathrm{HbA1c}$ concentrations were affected by moderate to severe lipaemia and by haemolysis. The reference interval for $\mathrm{HbA1c}$ was 9-18.5 mmol/ mol, with a mean of $14.3 \mathrm{mmol} / \mathrm{mol} \mathrm{HbA} 1 \mathrm{c}$ and a range of 9 to $19 \mathrm{mmol} / \mathrm{mol}$. There was no apparent effect of age or breed on $\mathrm{HbA} 1 \mathrm{c}$ concentration.

\section{CONCLUSIONS}

The assay evaluated provides a reliable method of canine $\mathrm{HbA1c}$ measurement with good analytical performance. Further work to assess the effect of interference from lipaemia and haemolysis is needed.

\section{HbA1c measurements in canine diabetic patients compared to normoglycaemic controls and fructosamine measurements.}

\section{Susanna Spence, Floortje Goemans, Aimee Hope, Ian Ramsey}

University of Glasgow, Glasgow, UK

\section{BACKGROUND}

Diabetes mellitus is a common endocrinopathy in small animal medicine yet complications such as diabetic ketoacidosis and cataracts frequently arise. Fructosamine levels and glucose curves are often used to evaluate insulin therapy in dogs but they are not without their limitations. In contrast, in human medicine, measurement of HbA1c (a form of glycosylated haemoglobin) is used to monitor diabetic stability by providing an indication of glycaemic control over the preceding 2 to 3 months. Moreover $\mathrm{HbA1c}$ concentrations are associated with outcome in human diabetes, whereas fructosamine values are not. Recently, the Siemens DCA Vantage $^{\circledR}$ analyser has been demonstrated to measure $\mathrm{HbA1c}$ reliably and repeatably in canine patients and a reference range has been identified.

\section{OBJECTIVE}

To compare HbA1c measurements in diabetic and non-diabetic patients within a hospital population and to determine the relationship between $\mathrm{HbA} 1 \mathrm{c}$ and fructosamine levels in diabetic patients.

\section{METHODS}

$\mathrm{HbA1c}$ was measured from EDTA samples using the Siemens DCA Vantage ${ }^{\circledR}$ analyser. Three groups were included in this study: an uncontrolled diabetic group (Group 1, $n=26$; which included 3 patients with concurrent hyperadrenocorticism); a normoglycaemic control group (Group 2, n=76); and a normoglycaemic group with hyperadrenocorticism (Group 3, n=5). All groups were age matched. Group 1 was 